\title{
A quantifiable approach in the comparison of isokinetic assessment data-new correlation equations for the Johnson antishear device and standard shin pad in the isokinetic assessment of the knee
}

\author{
CK Li, KM Chan, Stephen YC Hsu, P Chien and WN Wong \\ Department of Orthopaedics and Traumatology, Prince of Wales Hospital, Chinese University of Hong Kong, \\ Shatin, NT, Hong Kong
}

\begin{abstract}
The rehabilitation of the knee with a deficient anterior cruciate ligament (ACL) always presents a great challenge. Isokinetic assessment allows muscle performance to be quantified. The antishear device, introduced by Johnson, has the distinct advantage of minimizing the anterior translation force during isokinetic exercise. In a previous report, there was a significant difference between the Johnson double pad antishear device and the standard single pad device in knee extension. The main purpose of this study was to define a correlation equation such that data generated in the use of the two different devices can be compared for assessment. Ten subjects were tested with the Cybex isokinetic dynamometer with a randomly selected sequence of test speeds. Correlation coefficient $(r)$, regression analysis and paired $t$ tests $(P)$ were performed to estimate the correlation and difference between the two devices. It is concluded that a significant difference in the data generated between the two devices indicated that a single device should be selected to carry out a series of comparisons on a particular subject. However, if the two devices are used in a series of testings, a set of correlation equations has been defined to facilitate such comparison. The findings in this study will widen the application of comparison of isokinetic data in the quantitative approach of rehabilitation of the knee with a deficient anterior cruciate ligament.

(Br J Sports Med 1995; 29 : 171-173)
\end{abstract}

Keywords: isokinetic dynamometer; antishear device; standard shin pad; correlation

The rehabilitation after anterior cruciate ligament (ACL) injury or reconstruction surgery ${ }^{1-3}$ has long been a challenge to sports physicians and therapists. The therapeutic goal is to get the athlete back on the field as soon as possible. On the other hand, the chance of further injury needs to be reduced by an intensive rehabilitation protocol. Isokinetic exercise has proved to be one of the safest and most effective exercises in the

Address for correspondence: Professor KM Chan, Department of Orthopaedics and Traumatology, Prince of Wales Hospital, Chinese

University of Hong Kong, Shatin, NT, Hong Kong strengthening of muscle. The Cybex isokinetic dynamometer has been the pioneer testing and training machine for a long time. ${ }^{4-6}$ In 1982, Johnson ${ }^{7}$ modified the single pad device and introduced the new double pad anti-shear device for the rehabilitation and testing of knee injured patients, especially those patients with ACL problems. The advantage claimed was the reduction of anterior translation force needed during knee extension in patients with ACL insufficiency.

Several studies reported on the anti-shear property and the validity of this new double pad anti-shear device as an accurate test instrument. In 1982 Johnson $^{7}$ reported that anterior shearing force could be controlled by adjustment of the position between the two pads. Nisell et $a l^{8}$ calculated the tibiofemoral joint forces with a mathematical model. They reported that the torque magnitude of isokinetic extension was significantly lower with the resistance pad placed proximally rather than distally on the leg. The proximal positioning of the pad would reduce the anterior shear force but also promote a higher posterior directed shear force. Malone $^{9}$ recommended that the torque value generated in using the new double pad device could not be compared directly to that with the old single pad device. Timm ${ }^{10}$ reported that there was no significant difference between the single pad and the double pad device. He concluded that the anti-shear device effectively controlled anterior translation force during isokinetic exercise and that it was accurate and valid in isokinetic evaluation and exercise. Epler et $a l^{11}$ found that the quadriceps torque at low speed $\left(60^{\circ} \cdot \mathrm{s}^{-1}\right)$ and the total work at medium speed $\left(180^{\circ} \cdot \mathrm{s}^{-1}\right)$ were less with the use of the anti-shear device. In our previous study, ${ }^{12}$ we observed significant differences of torque, average work, and average power generated during the testing of the two devices.

The objective of this study was to generate correlation equations between the Johnson anti-shear device (double pad) and the standard shin pad (single pad). Most of the published papers on the Cybex used 
the standard shin pad as testing tool during isokinetic testing of the knee.

\section{Methods}

\section{Subjects}

Ten subjects (four male, six female) with no previous history of injury on either knee were recruited. The age ranged from 19-30 years (mean 25.2). All subjects were briefed on the operation of the testing device and how to provide maximum effort during the test. A consent form was signed by each subject before testing.

\section{Instrumentation}

Cybex $\mathrm{II}^{+}$dynamometer (Cybex, Division of Lumex Inc) was used with the HUMAC computer system (Computer Sports Medicine Inc) as the isokinetic testing machine. The evaluation of data output such as peak torque, average workload and average power were calculated by the HUMAC. Both the Cybex $\mathrm{II}^{+}$ dynamometer and the HUMAC were calibrated the day before the experiment.

\section{Procedure}

Each test was performed two speeds, slow speed $\left(60^{\circ} \cdot \mathrm{s}^{-1}\right)$ and medium speed $\left(180^{\circ} \cdot \mathrm{s}^{-1}\right)$. The testing speed used in each test was also randomized. Each subject was asked to do stretching exercises and $5 \mathrm{~min}$ on a static cycle before the test. At each test speed seven submaximal and two maximum contractions were performed as a warm up, with 1 min rest between each test speed. Five maximum contractions were assigned for each test speed.

The manufacturer's instruction on use of the Cybex II standard shin adapter (single pad) and the Johnson antishear device (double pad) were strictly followed. The single pad was placed such that the inferior border of the pad rested immediately superior to the medial malleolus. ${ }^{13}$ For the double pad, the superior border of the proximal pad was placed at the tibial tuberosity and the inferior border of the distal pad was placed immediately superior to the medial malleolus. The fulcrum was then positioned mid-way between the pads. ${ }^{9}$ All data were stored on the HUMAC.

Both knees of each subject were tested in this study. The 10 subjects were randomly divided into two groups. The first group used the single pad for the first testing and the double pad for the second testing after $3 \mathrm{~h}$ of rest. The second group repeated the same testing procedure but used the double pad for the first testing and the single pad for the second testing. As suggested by Epler $e t$ al, ${ }^{11}$ this would eliminate the bias of results due to the learning process of subjects. Among the 10 subjects, five were randomly selected to have their left knee as control. In these controls, a single pad was used on the first and second testing. This arrangement would reveal whether there was any difference between the first and second testing after $3 \mathrm{~h}$ of rest. As a result, we had 15 experimental records (10 right knee, five left knee) and five control records (five left knee).

\section{Data analysis}

The peak torque, average workload, and average power of knee extension and flexion were selected. Peak torque was measured in $\mathrm{Nm}$, average work in J, and average power in W. Paired $t$ tests were performed to determine the difference between the two devices. Correlation coefficient $(r)$ was computed to find out the correlation between the two devices. Regression analysis was executed to construct the correlation equations between the two devices.

\section{Results}

In the control group there was no significant difference in peak torque, average workload, or average power on both the extension and flexion moment between the two tests $(P>0.05)$. In the experimental group, a significant difference $(P<0.05)$ was observed in peak torque, average workload, and average power on knee extension between the two devices at both test speeds. However, there was no significant difference $(P>0.05)$ in knee flexion. The detailed results can be seen in our previously published study. ${ }^{12}$ In the experimental group, a very good correlation $(r>0.75)$ was seen in peak torque, average workload, and average power in both the extension and the flexion movement between the two devices. The correlation coefficients are shown in Table 1. A set of correlation equations (Table 2) was worked out for the differences between the double pad anti-shear device and single pad device. Those equations enabled us to convert the testing data that were used on the double pad anti-shear device to those used on the single pad device and vice versa.

\section{Discussion}

In our previously published study, ${ }^{12}$ we reported significant differences in the knee extension between the two devices in peak torque, average workload, and average power, but no significant difference in knee flexion. These results matched Nisell's ${ }^{8}$ and Epler's' $s^{11}$ findings. A detailed explanation and discussion can be seen in our published study. ${ }^{12}$

Timm ${ }^{10}$ reported good correlation in extension peak torque $(r=0.97)$, flexion peak torque $(r=0.99)$, extension average power, and flexion average power at both slow and medium speed. Similar findings were observed in our study. Good correlations in extension average workload and flexion average workload at both speeds were also obtained in this study.

A set of correlation equations on extension and flexion movement has been worked out for conversion of data between the double pad anti-shear device and the single pad device. Even though there was no significant difference between the two devices in flexion, the values for the single pad device were still greater than from the double pad device. For accuracy, we still recommend the use of correlation equations on flexion. These equations are very useful in the clinical situation. For example, two test records from the same patient are 
Table 1. The correlation coefficients ( $r$ ) (experimental group)

\begin{tabular}{lcc}
\hline Parameter & Extension & Flexion \\
\hline Slow speed & & \\
$\quad$ Peak torque & 0.9453 & 0.9254 \\
$\quad$ Average workload & 0.9031 & 0.9170 \\
$\quad$ Average power & 0.9336 & 0.9408 \\
Medium speed & & \\
$\quad$ Peak torque & 0.9406 & 0.8858 \\
$\quad$ Average workload & 0.9224 & 0.8646 \\
$\quad$ Average power & 0.9586 & 0.9047 \\
\hline
\end{tabular}

available, one using the standard shin pad before the surgical operation was performed, the other using the Johnson anti-shear device during rehabilitation. Since the double pad device was proved to decrease the antishearing force during knee exercise, and the device was also accurate and valid in isokinetic evaluation and exercise, the two tests could not be compared directly as stated by Malone. ${ }^{2}$ However, with these correlation equations, the data obtained from the Johnson antishear device could be converted to data corresponding to the standard shin pad and vice versa. The two tests, although using different devices, could still be compared. Apart from comparing data from the same patient, we would also like to compare the data between the norm of similar age groups. However, all the data from the norm were obtained with the use of the single pad device in the assessment of the knee. Again, with the use of the correlation equation the data can be compared. This is useful in that it can show patients whether they are as fit as average persons of a similar age.

In conclusion, significant differences in the results of isokinetic testing exist between the two devices. This indicates the importance of selecting a single device for patients or athletes during the whole series of tests. Direct comparison of results obtained from these two devices will lead to wrong conclusions.

The high correlation coefficient in peak torque, average workload, and average power in using the two devices suggest that the data from the Johnson antishear device and the standard shin pad can be compared by using the correlation equations. These equations are very useful in comparing test results within the same patient or among different subjects when the tests were performed with different devices.

\section{References}

1 Anderson C, Gillquist J. Treatment of acute isolated and combined ruptures of the anterior cruciate ligament-a long term follow up study. Am J Sports Med 1992; 20: 7-12.

2 Aglietti P, Buzzi R, D'Andria S, Zaccherotti G. Long-term study of anterior cruciate ligament reconstruction for chronic instability using the central one-third patellar tendon and a lateral extraarticular tenodesis. Am J Sports Med 1992; 20: 38-45.

3 Harner CD, Irrgang JJ, Paul J, Dearwater S, Fu FH. Loss of motion after anterior cruciate ligament reconstruction. Am J Sports Med 1992; 20: 499-506.

4 Sherman WM, Pearson DR, Plyley MJ, Costill DL, Habansky AJ, Vogelgesang DA. Isokinetic rehabilitation after surgery-a review of factors which are important for developing physiotherapeutic techniques after knee surgery. Am J Sports Med 1982; 10: 155-61.

5 Murray SM, Warren RF, Otis JC, Kroll M, Wickiewicz T. Torquevelocity relationships of the knee extensor and flexor muscles in individuals sustaining injuries of the anterior cruciate ligament. Am J Sports Med 1984; 12: 436-40.

6 Seto J, Orofino A, Morrissey M, Medeiros J, Mason W. Assessment of quadriceps/hamstring strength, knee ligament stability, functional and sports activity level five years after anterior cruciate ligament reconstruction. Am J Sports Med 1988; 16: $170-80$

7 Johnson D. Controlling anterior shear during isokinetic knee extension exercise. J Orthop Sports Phys Ther 1982; 4: 23-31.

8 Nisell R, Ericson M, Nemeth G, Ekholm J. Tibiofemoral joint force during isokinetic knee extension. Am J Sports Med 1989; 17: 49-55.

9 Malone T. Clinical use of the Johnson anti-shear device: how and why to use it. J Orthop Sports Phys Ther 1986; 7: 304-9.

10 Timm K. Validation of the Johnson anti-shear accessory of an accurate and effective clinical isokinetic instrument. J Orthop Sports Phys Ther 1986; 7 : 298-303.

11 Epler M, Nawaczenski D, Englehardt T. Comparison of the Cybex II standard shin adapter versus the Johnson anti-shear device in torque generation. J Orthop Sports Phys Ther 1988; 9: 284-86.

12 Li CK, Chan KM, Hsu SYC, Chien P, Wong MWN, Yuan Y. The Johnson anti-shear device and standard shin pad in the isokinetic assessment of the knee. Br J Sports Med 1993; 27: 49-52.

13 Isolated joint testing and exercise: $a$ handbook for using Cybex II and UBXT. Ronkonkoma, NY, USA: Cybex Division of Lumex, Inc, 1980.

Table 2. The correlation equations (experimental group)

\begin{tabular}{lll}
\hline Parameter & Extension & Flexion \\
\hline $\begin{array}{l}\text { Slow speed } \\
\text { Peak torque } \\
\text { Average workload }\end{array}$ & $\mathbf{J}=0.8861 \times \mathbf{S}+1.9740$ & $\mathbf{J}=0.9153 \times \mathbf{S}+3.0452$ \\
Average power & $\mathbf{J}=0.8169 \times \mathbf{S}-1.5419$ & $\mathbf{J}=0.8545 \times \mathbf{S}+1.1934$ \\
Medium speed & $\mathbf{J}=0.8989 \times \mathbf{S}-2.2603$ & $\mathbf{J}=0.9121 \times \mathbf{S}+2.1423$ \\
Peak torque & & $\mathbf{J}=1.2077 \times \mathbf{S}-4.0335$ \\
Average workload & $\mathbf{J}=1.0595 \times \mathbf{S}-4.5904$ & $\mathbf{J}=1.0170 \times \mathbf{S}-2.2666$ \\
Average power & $\mathbf{J}=1.0644 \times \mathbf{S}-8.9738$ & $\mathbf{J}=1.1445 \times \mathbf{S}-10.704$ \\
\hline
\end{tabular}

${ }^{*} \mathbf{J}=$ Johnson anti-shear device; $\mathbf{S}=$ standard shin pad 